\title{
Effect of Cross-linking on the Performance and Stability of Photocatalytic Photosystem I Films
}

Siyuan Yang ${ }^{a}$, Maxwell T. Robinson ${ }^{a}$, Faustin Mwambutsa $^{a}$, David E. Cliffel ${ }^{b}$, and G. Kane Jennings ${ }^{a^{*}}$

\begin{abstract}
Extracted from the chloroplasts of green plants, Photosystem I (PSI) is a multi-subunit protein complex that can be deposited as a thin film onto electrodes and incorporated into biohybrid photovoltaic devices to convert sunlight into usable energy. Previously, our group characterized persistent photocurrents over nine months using PSI multilayer films in an aqueous solution of redox mediators. However, the loss of periphery PSI proteins upon exposure to mediator solutions is known to affect the photocatalytic performance of the overall film. The cross-linker 2-iminothiolane (2IT) is capable of connecting protein molecules with covalent bonds, and is herein used to stabilize PSI multilayer films on gold substrates against significant desorption/degradation. Results showed that the thickness retainment of the cross-linked PSI films was superior to that of non-cross-linked films. PSI films modified by 2IT maintained production of photocurrent with long-term exposure to aqueous solutions in contrast to noncross-linked control films.
\end{abstract}

Keywords Protein, Photocatalysis, Solar Energy, Biohybrid, Photosynthesis ${ }^{1}$ 


\begin{abstract}
Abbreviations Photosystem I (PSI), 2-iminothiolane (2IT), glutaraldehyde (GA), terepththaldialdehyde (TPDA), 2-aminoethanethiol (AET), self-assembled monolayer (SAM), sodium L-ascorbate (Asc), 2,6-dichlorophenolindphenol (DCPIP)
\end{abstract}

\title{
1. Introduction
}

Photosynthesis is the most important solar energy conversion process on the planet, through which plants, algae, and cyanobacteria convert sunlight into stored energy in the form of reduced carbon.[1] Photosystem I (PSI), a $500 \mathrm{kDa}$ integral protein complex, is a chief driver of oxygenic photosynthesis, transducing solar irradiance with near-unity quantum yield.[2] Antenna chlorophylls located on the periphery of PSI simultaneously coordinate photon absorbance and resonant energy transfer to a special chlorophyll A dimer $\left(\mathrm{P}_{700}\right)$, which generates a high-energy electron. An internal electron transfer chain guides this electron to a terminal iron-sulfur acceptor $\left(\mathrm{F}_{\mathrm{B}}\right)$ in about 10-30 ps.[3] Extracted from plants or cyanobacteria and assembled as a thin film atop an electrode, PSI can be harnessed to photocatalyze electron transfer between an electrode and solution-phase mediators with appropriate formal potentials.[4] Due to the rapid charge separation and internal quantum efficiency of PSI, researchers are actively investigating its incorporation into highly sustainable biohybrid photovoltaic devices.[5-7]

Researchers have made several key advances to integrate PSI and related biomolecules with non-biological systems for the design of more efficient photoelectrochemical cells.[8-15] Mershin et al. designed a low-cost PSI biophotovoltaic solar cell device by stabilizing dry PSI via surfactant peptides self-assembled on nanostructured semiconductors, including mesoporous $\mathrm{TiO}_{2}$ and $\mathrm{ZnO}$ nanowires.[11] Yu et. al. explored the hierarchical design of PSI-based dye- 
sensitized solar cells using screenprinted meso- and macroporous $\mathrm{TiO}_{2}$ films.[16] Kothe et al.[9] integrated PSI in an Os-based redox hydrogel to efficiently route cathodic current through the protein that enabled electron turn over rates almost 7x higher than PSI in nature. Our group has deposited PSI on a variety of electrodes to investigate photocurrent generation. We reported a method to assemble multilayer PSI films with controlled thickness ranging from $0.4-3 \mu \mathrm{m}$ based on the number of drop-casting deposition steps.[17] We observed that thicker PSI films on electrodes achieved larger photocurrents because they increase light absorption and provide a high concentration of sites near the electrode surface where oxidation and reduction of electrochemical mediators may occur. In addition, LeBlanc et al. immobilized $\sim 1$ - $\mu$ m-thick PSI films onto p-doped silicon and observed an 80-fold photocurrent enhancement relative to that produced by films of similar thickness on gold under similar conditions.[14] This strong enhancement is aided by favorable band alignment between silicon and PSI that enables unidirectional electron flow from the electrode into the mediator-rich protein film.

Although thicker PSI films generally yield higher photocurrents,[17] the stability of the current must be taken into consideration. Previously, we tested the stability of a PSI prototype "wet" cell over a period of 280 days by keeping the cell in the dark and testing it periodically in light.[13] This approach was taken to investigate if PSI could remain functional over periods of time that extend well beyond the normal growing season of the source plant. After the photocurrent reached its peak, it decreased by $50 \%$ on the following day and remained nearly constant for the remainder of the 9-month testing period, generating stable photocurrent during this time. The decrease in photocurrent was attributed to some desorption and/or degradation of a fraction of PSI complexes that were not well stabilized in the aqueous mediator solution. Since PSI exhibits limited water solubility, minimizing desorption of PSI into aqueous mediator 
solutions is critical to maintain performance in these photoelectrochemical devices. Further, as PSI is now being investigated in a broader array of solar energy conversion systems, including solid-state devices[18-20] with more aggressive solution-based processing and as composite films,[15] methods to stabilize the complex within these systems will become increasingly important. Here we seek to examine the robustness of PSI multilayer films upon exposure to aqueous solutions and develop a straightforward method to stabilize them.

Robust electrode systems based on PSI multilayer films have two basic requirements. First, the thickness or loading of the protein on the electrode and the photoresponse of the PSI multilayer films should be maintained. Second, the electrode interface should be stable and resistant to oxidation. We have selected highly stable gold substrates in this study, allowing us to directly focus on the stability of the PSI films without concern for degradation of the electrode surface.

To improve the stability of PSI films, we have investigated the ability to cross-link adjacent PSI proteins via covalent bonds within multilayer PSI films on electrode substrates. Initially, we examined three cross-linkers, including 2-iminothiolane (2IT, Traut's reagent), glutaraldehyde (GA), and terepththaldialdehyde (TPDA). TPDA showed evidence of crosslinking but produced an insoluble precipitate on the protein film; GA cross-linked the protein well to boost stability but decreased the photocatalytic performance of the protein. Therefore, we focused our research on the use of 2IT as a cross-linker to stabilize PSI films on gold substrates. 2IT can react with terminal lysines or between a lysine and cysteine to crosslink the protein (Scheme I).[21] Additionally, 2IT provides a covalent linkage between the amineterminated self-assembled monolayer prepared from 2-aminoethanethiol (AET) and the first layer of proteins deposited on the surface. The thickness, topography, and electrochemical 
properties of PSI films were analyzed before and after exposure to aqueous buffer solutions to assess the performance of 2IT as a cross-linker.

\section{Materials and Methods}

2.1. Materials. Materials were purchased as follows: $\mathrm{KCl}, 2$-iminothiolane $\bullet \mathrm{HCl}$ (2IT), and 2-aminoethanethiol (AET) from Sigma-Aldrich; sodium L-ascorbate (Asc) from Acros; 2,6dichlorophenolindphenol (DCPIP) from Encompass Chemicals. $\mathrm{N}_{2}$ was purchased from A-L Compressed Gases. Deionized water (16.7

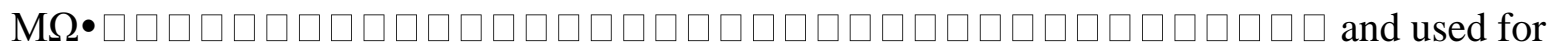
rinsing samples and substrates and for preparation of PSI solutions. Gold shot (99.99\%) was purchased from J\&J Materials. Chromium-coated tungsten filaments were obtained from R.D. Mathis. Silicon (100) wafers (p-doped) were purchased from University Wafers (Boston, MA). An electrochemical sample mask made with plater's tape was purchased from Gamry Instruments. Ethanol was obtained from Decon Labs, Inc. Fresh organic spinach was purchased from a nearby grocery store (Harris Teeter).

\subsection{Preparation of Gold Substrates and Self-Assembled Monolayers (SAMs). Gold-}

coated silicon substrates were used as the electrodes in all experiments. To prepare gold-coated silicon, p-doped silicon (100) wafers were rinsed with ethanol and deionized water, then dried with $\mathrm{N}_{2}$, before being placed into a metal atom evaporator. Chromium (100 $\left.\mathrm{A}\right)$ and $\mathrm{Au}(1250 \AA$ ) were evaporated in sequence onto the wafers at a rate of $1-2 \AA / s$ in a diffusion-pumped chamber with a base pressure of $4 \times 10^{-6}$ torr. After removal from the evaporation chamber, the substrates were cut into $1.2 \mathrm{~cm}$ x $3.5 \mathrm{~cm}$ pieces. Self-assembled monolayers (SAMs) on gold surfaces were prepared by exposing the gold substrates to $1 \mathrm{mM}$ ethanolic solutions of 2-aminoethanethiol 
(AET) for $1 \mathrm{~h}$. Then, an electrochemical mask (Gamry Instruments) was placed onto the SAMcoated substrate to create a $0.2 \mathrm{~cm}^{2}$ exposed circular area for the deposition of a PSI film.

2.3. Photosystem I Extraction and Isolation. PSI was extracted from commercial baby spinach leaves by the method of Reeves and Hall with some adaptations.[22, 23] The thylakoid membrane was removed from the suspension of spinach by centrifugation. PSI complexes were separated from the thylakoid membrane by additional centrifugation. The PSI suspension was purified using a hydroxylapatite column. The PSI effluent was collected into microcentrifuge tubes and dialyzed (1 mL PSI solution: $2 \mathrm{~L}$ deionized water) using a 10000 MW cutoff membrane for $24 \mathrm{~h}$. After dialysis, the concentration of the extracted PSI solution was approximately $1.3 \mu \mathrm{M}$, and the number of externally bound chlorophylls per PSI complex was $\sim 70$, which was determined by UV-Vis analysis.[24]

2.4. Cross-linked PSI Multilayer Assembly. PSI multilayer films with and without 2IT cross-linking were prepared by the vacuum-assisted drop-casting method.[25] $50 \mu \mathrm{L}$ of PSI solution $(1.3 \mu \mathrm{M})$ was pipetted onto the exposed area of the SAM-coated gold substrate through the electrochemical mask. The vacuum chamber was sealed and pumped down to a pressure of $30 \mathrm{mTorr}$ for $30 \mathrm{~min}$ to evaporate water from the PSI solution. Pure PSI complexes tend to aggregate on the electrode since PSI is the least soluble component in the solution. To cross-link PSI films, $50 \mu \mathrm{L}$ of $1 \mathrm{mM}$ 2IT aqueous solution was added directly atop the dried PSI multilayer films (Figure 1). The vacuum-assisted method was applied again to allow water to evaporate and cross-linker molecules to penetrate through the PSI films. The films were rinsed with DI water afterwards.

2.5. Profilometry. A Veeco DEKTAK 150 surface profilometer was used to measure the thickness of PSI multilayer films. A cut was made by hand through the PSI multilayer films 
down to the substrate using forceps so that the bottom line of the valley shows the bare substrate. The measurements were taken over a distance of $800 \mu \mathrm{m}$ using a stylus with a $12.5 \mu \mathrm{m}$ radius, applying $1 \mathrm{mg}$ force, and employing a hills-and-valleys detection mode. The reported thickness values and errors represent the averages and standard deviations, respectively, of at least 5 independently prepared films.

2.6. Electrochemical Impedance Spectroscopy (EIS). Electrochemical impedance spectra were collected by a Gamry Reference 6000 electrochemical workstation in a three-electrode electrochemical cell. Gold substrates with PSI films were used as working electrodes, and a platinum mesh was used as the counter electrode, with a $\mathrm{Ag} / \mathrm{AgCl}$ reference electrode. An aqueous solution of $1 \mathrm{mM}$ DCPIP/25 mM sodium ascorbate and $100 \mathrm{mM} \mathrm{KCl}$ served as the redox electrolyte. All data were collected at cell open circuit potential $(0.1 \mathrm{~V}$ vs. $\mathrm{Ag} / \mathrm{AgCl})$ in the range of $10^{-2}$ to $10^{4} \mathrm{~Hz}$ with 10 points per decade and a voltage amplitude of $5 \mathrm{mV}$. A Randles circuit model[26],[27] was fit to the data to determine the resistance and capacitance of the films.

\subsection{Photochronoamperometric Measurement. Photocatalytic performance of PSI}

multilayer films immobilized on gold substrates was evaluated using photochronoamperometry with $20 \mathrm{~s}$ of white light illumination. Experiments were performed using a $\mathrm{CH}$ instruments CHI660a electrochemical workstation under ambient conditions and a three-electrode electrochemical cell, consisting of $1 \mathrm{mM}$ DCPIP/ $25 \mathrm{mM}$ sodium ascorbate mediators in a 100 $\mathrm{mM} \mathrm{KCl} \mathrm{(aq)} \mathrm{supporting} \mathrm{electrolyte.} \mathrm{Working} \mathrm{electrodes} \mathrm{consisted} \mathrm{of} \mathrm{PSI} \mathrm{multilayers} \mathrm{atop} \mathrm{an}$ AET-decorated gold surface. A platinum mesh was used as the counter electrode and an $\mathrm{Ag} / \mathrm{AgCl}$ electrode was used as the reference electrode. The photo response provided in this section was generated by illuminating the sample with white light from a Leica KL 2500LCD 
lamp at an approximate light intensity of $140 \mathrm{~mW} / \mathrm{cm}^{2}$. All photocurrent measurements were performed at the cell open circuit potential $(0.1 \mathrm{~V}$ vs. $\mathrm{Ag} / \mathrm{AgCl})$.

2.8. Quartz Crystal Microbalance in Dissipation Mode (QCM-D). QCM in dissipation mode (QCM-D) was performed in a flowing aqueous solution of $0.1 \mathrm{M} \mathrm{KCl}(\mathrm{aq})$ with a Q-Sense E4 to measure the change in both frequency and dissipation of the PSI film before and after crosslinking. By applying the Voigt viscoelastic model with the Qtool ${ }^{\circledR}$ software, the shear modulus of each film was determined.

\subsection{Polarized Modulation Infrared Reflectance-Absorption Spectroscopy (PM-IRRAS).}

PM-IRRAS spectra were collected with a Bruker Tensor 26 infrared spectrometer equipped with a liquid-nitrogen cooled mercury-cadmium-telluride (MCT) detector and a Hinds Instruments PEM-90 photoelastic modulator. The source beam used a half wavelength $(\lambda / 2)$ retardation modulated at a frequency of $50 \mathrm{kHz}$ and set at $80^{\circ}$ incident to the sample surface. Spectra were collected over $12 \min$ (466 scans) at a resolution of $2 \mathrm{~cm}^{-1}$. For the PSI film, particular attention was paid to the Amide I $\left(1600-1700 \mathrm{~cm}^{-1}\right)$ and Amide II $\left(\sim 1500-1600 \mathrm{~cm}^{-1}\right)$ stretching vibrational bands to assess if cross-linking had altered the secondary structure of the PSI proteins within the film. The Amide I peak was deconvoluted with a Lorentzian curve-fitting model into several minor peaks that represent elements of secondary structure, including $\beta$ turn (1616 1620 and 1680-1690 $\left.\mathrm{cm}^{-1}\right), \beta$ sheet $\left(1624-1636\right.$ and $\left.1692-1697 \mathrm{~cm}^{-1}\right), \alpha$ helix $(1648-1660$ and $\left.1663-1667 \mathrm{~cm}^{-1}\right)$, and unordered helix $\left(1638-1645 \mathrm{~cm}^{-1}\right)$.[28-33] The deconvolution was accomplished with Curve Fitting C, free software at http://terpconnect.umd.edu/ toh/spectrum/CurveFittingC.html.

\section{Results and Discussion}




\subsection{Effect of Cross-linking on Thickness and Mechanical Properties. To cross-link PSI}

films, $50 \mu \mathrm{L}$ of a $1 \mathrm{mM} 2 \mathrm{IT}$ aqueous solution was drop-cast onto the surface of a PSI film, wetting it well, before vacuum was applied to gradually remove residual water. The amount of 2IT added to the PSI surface was sufficient to increase the film thickness by $300 \mathrm{~nm}$ if it formed an insoluble precipitate atop the protein film. However, no precipitate was observed, and film thicknesses by profilometry (Table 1) were statistically similar for the cross-linked films and non-cross-linked control films. These observations indicate that the cross-linkers penetrate the PSI film.

QCM in dissipation mode (QCM-D) was employed at different overtones to assess the effect of cross-linking on film stiffness by placing the substrate in a flowing aqueous buffer solution after each step of PSI film deposition and crosslinking. Figure 2(a) shows that the measured frequency decreases after PSI is deposited, consistent with an increase in mass on the surface of the crystal. However, after 2IT is added, the frequency increases ( $-\Delta f$ decreases). The increase in frequency here is consistent with a stiffer film that is less coupled to the contacting aqueous solution, given that a known mass has been added to the film. In agreement with this interpretation, film dissipation increases after PSI is deposited but decreases after the cross-linker is added (Figure 2(b)). By applying the Voigt viscoelastic model for these measurements from the Qtool ${ }^{\circledR}$ software, the shear modulus of the reference PSI film is estimated as $3.2 \mathrm{kPa}$ while that for the 2IT-cross-linked film is nearly twice as high at $6.1 \mathrm{kPa}$. The higher shear modulus indicates that the cross-linked film is more rigid than the reference film, which is consistent with interprotein cross-linking.

\subsection{Effect of Cross-linking on Secondary Structure and Barrier Properties. PSI}

multilayer films were characterized by PM-IRRAS (Figure S1; Supporting Information) before 
and after cross-linking to assess whether the cross-linker caused any structural changes to the protein film. An examination of the entire spectrum $\left(700-4000 \mathrm{~cm}^{-1}\right)$ revealed no new peaks after cross-linking. 2IT is expected to react and add $\mathrm{C}-\mathrm{N}$ bonds within the film, but the added absorbance of these bonds cannot be distinguished from the large background of $\mathrm{C}-\mathrm{N}$ bonding in the protein. In addition, the reaction of 2IT would add disulfide bonds, but these are generally weak and appear at $<600 \mathrm{~cm}^{-1}$,[34] beyond the spectral window. To examine indirect effects of cross-linker, the secondary structure of the PSI film before and after cross-linking was determined by deconvolution of the Amide I absorption peak (Table 2). The cross-linked film displays similar composition of $\beta$-sheet and unordered helix as the control, but lower $\alpha$-helix and higher $\beta$-turn percentages. These changes are small, considering the overall error involved in the deconvolution $(12 \%)$.

We have used EIS to assess the role of the cross-linker in limiting the transfer of aqueous ions (1 mM DCPIP/25 mM Asc) to the electrode surface (Figure 3). The impedance for the cross-linked film is slightly higher than that for the non cross-linked film at intermediate frequencies where capacitance is determined and 3-4 times higher at low frequencies where the resistance against ion transfer is probed. These spectra are very similar to controls of the SAM and the cross-linked SAM in which no PSI film is present (Figure S2; Supporting Information), indicating that the impedance provided by the PSI film is insignificant on the scale of the spectra. Further, the fact that no intermediate time constant exists in the spectra of Figure 3 indicates that ion transfer through these PSI films is without measurable impedance, which is expected if the protein film is saturated with aqueous solution. Therefore, in interpreting the impedance spectra, the resistance at high frequency is due to ion transfer in solution, and that at low frequency is the resistance to charge transfer at the metal interface, which is affected by the SAM and its 
interaction with the protein film. The capacitive response at intermediate frequencies is due to effects at the Au/SAM/PSI interface and not influenced by the protein film.

The impedance spectra in Figure 3 were fit with a Randles equivalent circuit model[26],[27] to assess the effects of cross-linker on resistances and capacitance. The charge transfer resistance $\left(R_{\mathrm{ct}}\right)$ of the cross-linked film is 3.3 times greater than the control film, and the interfacial capacitance of the cross-linked film is only about half of that of the control film (Table 1). These results indicate that the cross-linker affects ion transfer primarily at the SAM/protein interface where cross-linking between the SAM and protein limit the approach of ions to the electrode. The lower interfacial capacitance is consistent with a thicker interfacial film due to the cross-linker being anchored onto the SAM and likely bridging to the nearby proteins. To summarize, addition of cross-linker increases impedance to ion transfer near the electrode interface but does not present a measurable barrier to ion transfer through the protein film.

3.3. Effect of Cross-linkers on Stability. To investigate the ability of cross-linkers to prevent PSI proteins within films from desorbing into solution, films with and without crosslinkers were exposed to $100 \mathrm{mM} \mathrm{KCl} \mathrm{(aq)} \mathrm{for} \mathrm{up} \mathrm{to} 72 \mathrm{~h}$, and their thicknesses were measured before and after exposure via profilometry. The thicknesses of the films were $1200+/-100 \mathrm{~nm}$ before exposure to aqueous solution. Figure 4 compares the percentage of thickness remaining after different times of exposure to the aqueous solution for a non-cross-linked control film and a film cross-linked with 2IT. Both films exhibited some loss of PSI in the first $8 \mathrm{~h}$. The crosslinked PSI films lost $\sim 15 \%$ of film thickness in the first $8 \mathrm{~h}$ of exposure, but very little after that, maintaining $75-80 \%$ of the original film after 3 days of exposure. In contrast, the control films 
lost over $50 \%$ in the first $8 \mathrm{~h}$ and more than $60 \%$ after 3 days of exposure. The 2IT cross-linker effectively stabilizes PSI complexes in these films to reduce desorption by $\sim 3$ fold.

Photochronoamperometry was employed to determine the effect of cross-linker on the photocurrent generation by PSI films. Here, we used dichlorophenolindophenol (DCPIP) as the electron acceptor and sodium ascorbate as a sacrificial electron donor in photochronoamperometric experiments, with an illumination time of $20 \mathrm{~s}$. When the films are irradiated by light, photocurrent is generated, as the PSI complexes generate oxidized and reduced species near the gold electrode through electron transfer from/to redox mediators in solution. As shown in Figure 5(a), the photocurrents generated by the non cross-linked control film and the film cross-linked by 2IT are nearly identical before exposure to the $100 \mathrm{mM} \mathrm{KCl}$ (aq) solution. This result suggests that cross-linking with 2IT does not damage the electrontransfer chain within PSI or prevent electron transfer with mediators. The fact that impedance results for the cross-linked and control films are different but the photocurrents are similar suggests that the photocurrents are not strongly affected by the increased impedance near the electrode surface.

Figure 5(b) shows the photocurrent generation of PSI films with/without cross-linker after exposure to $100 \mathrm{mM} \mathrm{KCl} \mathrm{(aq)} \mathrm{for} 3$ days to assess the photocurrent stability of these films. The photocurrent generated by the PSI films cross-linked by 2IT is similar both before and after exposure, while that generated by the control films decreased by $2-3$ fold. The greater amount of PSI lost from the non crossed-linked films upon exposure to aqueous solution (Figure 4) is consistent with the greater decrease in photocurrent, as we have previously shown that PSI film thickness and photocurrent are positively correlated.[17] The slight loss of thickness in 2ITstabilized PSI films (Figure 4) does not result in measurable loss in photocurrent (Figure 5(b)), 
indicating that the small amount of protein loss was not integral to photocurrent production by the film.

\section{Conclusions}

2IT covalently cross-links a thin PSI multilayer film without significantly altering film thickness or protein secondary structure but affecting other film properties. The use of 2IT results in an increased protein film stiffness, based on a $2 x$ increase in the shear modulus of the film.

Crucially, the cross-linker reduces by $\sim 3 x$ the amount of protein desorbed from the surface into an aqueous solution after 3 days. Most important, cross-linking with 2IT does not affect the photocurrent generated by the film either before or after exposure to aqueous solutions for 3 days.

\section{Acknowledgments}

We gratefully acknowledge financial support from the United States Department of Agriculture [grant number 2013-67021-21029 USDA]; NSF EPSCoR [grant number EPS 1004083]; the National Science Foundation [grant number DMR-1507505]; and the Scialog Program from the Research Corporation for Science Advancement. 


\section{References}

[1] R.E. Blankenship, D.M. Tiede, J. Barber, G.W. Brudvig, G. Fleming, M. Ghirardi, M.R. Gunner, W. Junge, D.M. Kramer, A. Melis, T.A. Moore, C.C. Moser, D.G. Nocera, A.J. Nozik, D.R. Ort, W.W. Parson, R.C. Prince, R.T. Sayre, Comparing Photosynthetic and Photovoltaic Efficiencies and Recognizing the Potential for Improvement, Science, 332 (2011) 805-809.

[2] A.N. Amunts, N. Nelson, O. Drory, The Structure of Plant Photosystem I at 3.4 Angstrom Resolution, Photosynthesis Research, 91 (2007) 151-159.

[3] L. Frolov, O. Wilner, C. Carmeli, I. Carmeli, Fabrication of Oriented Multilayers of Photosystem I Proteins on Solid Surfaces by Auto-Metallization, Advanced Materials, 20 (2008) 263-266.

[4] M. Ciobanu, H.A. Kincaid, V. Lo, A.D. Dukes, G. Kane Jennings, D.E. Cliffel, Electrochemistry and photoelectrochemistry of photosystem I adsorbed on hydroxylterminated monolayers, Journal of Electroanalytical Chemistry, 599 (2007) 72-78.

[5] A. Badura, T. Kothe, W. Schuhmann, M. Rogner, Wiring Photosynthetic Enzymes to Electrodes, Energy and Environmental Science, 4 (2011) 3263-3274.

[6] C.E. Lubner, R. Grimme, D.A. Bryant, J.H. Golbeck, Wiring Photosystem I for Direct Solar Hydrogen Production, Biochemistry, 49 (2010) 404-414.

[7] C.E. Lubner, P. Knorzer, P.J.N. Silva, K.A. Vincent, T. Happe, D.A. Bryant, J.H. Golbeck, Wiring an [FeFe]-Hydrogenase with Photosystem I for Light-Induced Hydrogen Production, Biochemistry, 49 (2010) 10264-10266.

[8] J.D.J. Olmos, J. Kargul, A quest for the artificial leaf, International Journal of Biochemistry \& Cell Biology, 66 (2015) 37-44.

[9] T. Kothe, S. Poller, F.Y. Zhao, P. Fortgang, M. Rogner, W. Schuhmann, N. Plumere, Engineered Electron-Transfer Chain in Photosystem 1 Based Photocathodes Outperforms Electron-Transfer Rates in Natural Photosynthesis, Chemistry-a European Journal, 20 (2014) 11029-11034.

[10] H. Yaghoubi, E. Lafalce, D. Jun, X.M. Jiang, J.T. Beatty, A. Takshi, Large Photocurrent Response and External Quantum Efficiency in Biophotoelectrochemical Cells Incorporating Reaction Center Plus Light Harvesting Complexes, Biomacromolecules, 16 (2015) 11121118.

[11] A. Mershin, K. Matsumoto, L. Kaiser, D. Yu, M. Vaughn, M.K. Nazeeruddin, B.D. Bruce, M. Graetzel, S. Zhang, Self-assembled Photosystem-I Biophotovoltaics on Nanostructured TiO2 and $\mathrm{ZnO}$, Scientific Reports, 2 (2012) 234.

[12] A.K. Manocchi, D.R. Baker, S.S. Pendley, K. Nguyen, M.M. Hurley, B.D. Bruce, J.J. Sumner, C.A. Lundgren, Photocurrend Generation from Surface Assembled Photosystem I on Alkanethiol Modified Electrodes, Langmuir, 29 (2013) 2412-2419.

[13] P.N. Ciesielski, F.M. Hijazi, A.M. Scott, C.J. Faulkner, L. Beard, K. Emmett, S.J. Rosenthal, D. Cliffel, G.K. Jennings*, Photosystem I-Based Biohybrid Photoelectrochemical Cells, Bioresource Technology 101 (2010) 3047-3053.

[14] G. LeBlanc, G. Chen, E.A. Gizzie, G.K. Jennings, D.E. Cliffel, Enhanced Photocurrents of PSI Films on p-Doped Silicon, Adv. Mater., 24 (2012) 5959-5962.

[15] G. LeBlanc, E. Gizzie, G.K. Jennings, D.E. Cliffel, Integration of Photosystem I with Graphene Oxide for Photocurrent Enhancement, Advanced Energy Materials, 4 (2014) 1301953. 
[16] D. Yu, M. Wang, G. Zhu, B. Ge, S. Liu, F. Huang, Enhanced photocurrent production by bio-dyes of photosynthetic macromolecules on designed TiO2 film, Scientific Reports, 5 (2015) 9375.

[17] P.N. Ciesielski, C.J. Faulkner, M.T. Irwin, J.M. Gregory, N.H. Tolk, D.E. Cliffel, G.K. Jennings, Enhanced Photocurrent Production by Photosystem I Multilayer Assemblies, Adv. Funct. Mater., 20 (2010) 4048-4054.

[18] P.I. Gordiichuk, G.-J.A.H. Wetzelaer, D. Rimmerman, A. Gruszka, J.W.d. Vries, M. Saller, D.A. Gautier, S. Catarci, D. Pesce, S. Richter, P.W.M. Blom, A. Herrmann, Solid-State Biophotovoltaic Cells Containing Photosystem I, Advanced Materials, 26 (2014) 48634869.

[19] E.A. Gizzie, J.S. Niezgoda, M.T. Robinson, A.G. Harris, G.K. Jennings, S.J. Rosenthal, D.E. Cliffel, Photosystem I-polyaniline/TiO2 solid-state solar cells: simple devices for biohybrid solar energy conversion, Energy \& Environmental Science, 8 (2015) 3572-3576.

[20] J.C. Beam, G. LeBlanc, E.A. Gizzie, B.L. Ivanov, D.R. Needell, M.J. Shearer, G.K. Jennings, C.M. Lukehart, D.E. Cliffel, Construction of a Semiconductor Biological Interface for Solar Energy Conversion: p-Doped Silicon/Photosystem I/Zinc Oxide, Langmuir, 31 (2015) 10002-10007.

[21] G. Bulaj, Formation of disulfide bonds in proteins and peptides, Biotechnology Advances, 23 (2005) 87-92.

[22] S.G. Reeves, D.O. Hall, Detergent Method for Extraction of Photosystem I, Methods in Enzymology, 69 (1980) 85-90.

[23] M. Ciobanu, H.A. Kincaid, G.K. Jennings, D. Cliffel, Directed Adsorption of Photosystem I on Micropatterned Surfaces, Langmuir, 21 (2005) 692-698.

[24] K. Baba, S. Itoh, G. Hastings, S. Hoshina, Photoinhibition of PSI Electron Transfer Activity in Isolated PSI Preparations wiht Different Chlorophyll Contents, Photosynthesis Research, 47 (1996) 121-130.

[25] P.N. Ciesielski, A.M. Scott, C.J. Faulkner, B.J. Berron, D.E. Cliffel, G.K. Jennings, Functionalized Nanoporous Gold Leaf Electrode Films for the Immobilization of Photosystem I, ACS Nano, 2 (2008) 2465-2472.

[26] A.J. Bard, L.R. Faulkner, Electrochemical Methods: Fundamentals and Applications, 2nd ed., Wiley, New York, 2001.

[27] G.K. Jennings, J.C. Munro, T.-H. Yong, P.E. Laibinis, Effect of Chain Length on the Protection of Copper by n-Alkanethiols, Langmuir, 14 (1998) 6130-6139.

[28] S. Seshadri, R. Khurana, A.L. Fink, Fourier Transform Infrared Spectroscopy in Analysis of Protein Deposits, Methods in Enzymology, Academic Press, New York, 1999, pp. 559-576.

[29] X. Ruan, J. Wei, Q. Xu, J. Wang, Y. Gong, X. Zhang, T. Kuang, N. Zhao, Comparison of the Effects of Triton X-100 Treatment on the Protein Secondary Structure of Photosystem I and Photosystem II Studied by FTIR Spectroscopy, J. Molecular Structure, 525 (2000) 97106.

[30] J.T. Pelton, L.R. McLean, Spectroscopic Methods for Analysis of Protein Secondary Structure, Analytical Biochemistry, 277 (2000) 167-176.

[31] J.L.R. Arrondo, F.M. Goñi, Structure and dynamics of membrane proteins as studied by infrared spectroscopy, Progress in Biophysics and Molecular Biology, 72 (1999) 367-405.

[32] N.P. Camacho, S. Rinnerthaler, E.P. Paschalis, R. Mendelsohn, A.L. Boskey, P. Fratzl, Complementary information on bone ultrastructure from scanning small angle X-ray scattering and Fourier-transform infrared microspectroscopy, Bone, 25 (1999) 287-293. 
[33] T. Heimburg, J. Schünemann, K. Weber, N. Geisler, FTIR-Spectroscopy of Multistranded Coiled Coil Proteins, Biochemistry, 38 (1999) 12727-12734.

[34] N. Biswas, A.J. Waring, F.J. Walther, R.A. Dluhy, Structure and Conformation of the Disulfide Bond in Dimeric Lung Surfactant Peptides SP-B1-25 and SP-B8-25, Biochemica et Biophysica Acta-Biomembranes, 1768 (2007) 1070-1082. 


\section{Table Captions}

Table 1. Profilometric film thickness, interfacial capacitance, and charge transfer resistance for the deposition of PSI and subsequent cross-linking with 2IT.

Table 2. Secondary structural elements as determined by deconvolution of the Amide I band using PM-IRRAS. Errors related to fitting the spectra are $12 \%$ of each reported value.

\section{Figure Captions}

Fig. 1. Process of preparing cross-linked PSI films on a SAM prepared from AET.

Fig. 2. QCM-D of a PSI film before and after cross-linking by 2IT. Experiments were performed in an aqeuous solution of $0.1 \mathrm{M} \mathrm{KCl}$. Values on the y axes express changes in (a) resonant frequency $(-\Delta f)$ and (b) dissipation, as referenced to the value of the bare crystal.

Fig. 3. Electrochemical impedance spectra of PSI films on gold before (Control) and after crosslinking with 2IT. The electrolyte solution consists of $1 \mathrm{mM} \mathrm{DCPIP/25} \mathrm{mM} \mathrm{sodium} \mathrm{ascorbate,}$ and $0.1 \mathrm{M} \mathrm{KCl}$. The spectra were fit with a Randles model equivalent circuit to determine charge transfer resistance and interfacial capacitance (Table 1), and the fits are shown as solid curves.

Fig. 4. Effect of cross-linker on PSI film stability. The percentage of film remaining, as determined via profilometric thickness, is shown after exposure to $100 \mathrm{mM} \mathrm{KCl}$ (aq) for the times indicated. The films investigated include PSI before (Control) and after cross-linking with 2IT.

Fig. 5. Effect of cross-linker on PSI photoelectrochemical performance. Photocurrent density of cross-linked and control PSI films are shown (a) before and (b) after 72-h exposure to $100 \mathrm{mM}$ $\mathrm{KCl}$ (aq). The film was in the dark until $20 \mathrm{~s}$, at which time the light was turned on. The light was turned off at $40 \mathrm{~s}$. 


\section{Tables}

Table 1

\begin{tabular}{lccc} 
Film & $\begin{array}{c}\text { Thickness } \\
(\mathrm{nm})\end{array}$ & $\begin{array}{c}\text { Capacitance } \\
\left(\mu \mathrm{F} / \mathrm{cm}^{2}\right)\end{array}$ & $\begin{array}{c}\text { Resistance } \\
\left(\mathrm{k} \Omega \mathrm{cm}^{2}\right)\end{array}$ \\
\hline PSI & $1210 \pm 240$ & $21 \pm 2$ & $30 \pm 12$ \\
PSI + 2IT & $1090 \pm 200$ & $11 \pm 3$ & $98 \pm 18$
\end{tabular}

Table 2

Structural Element $\quad$ PSI(Control) 2IT Cross-linked

\begin{tabular}{l|cc}
\hline$\alpha$-Helix (\%) & 30 & 24 \\
$\beta$-Sheet (\%) & 38 & 39 \\
Unordered Helix (\%) & 13 & 14 \\
$\beta$-Turn (\%) & 19 & 23
\end{tabular}


Figure 1

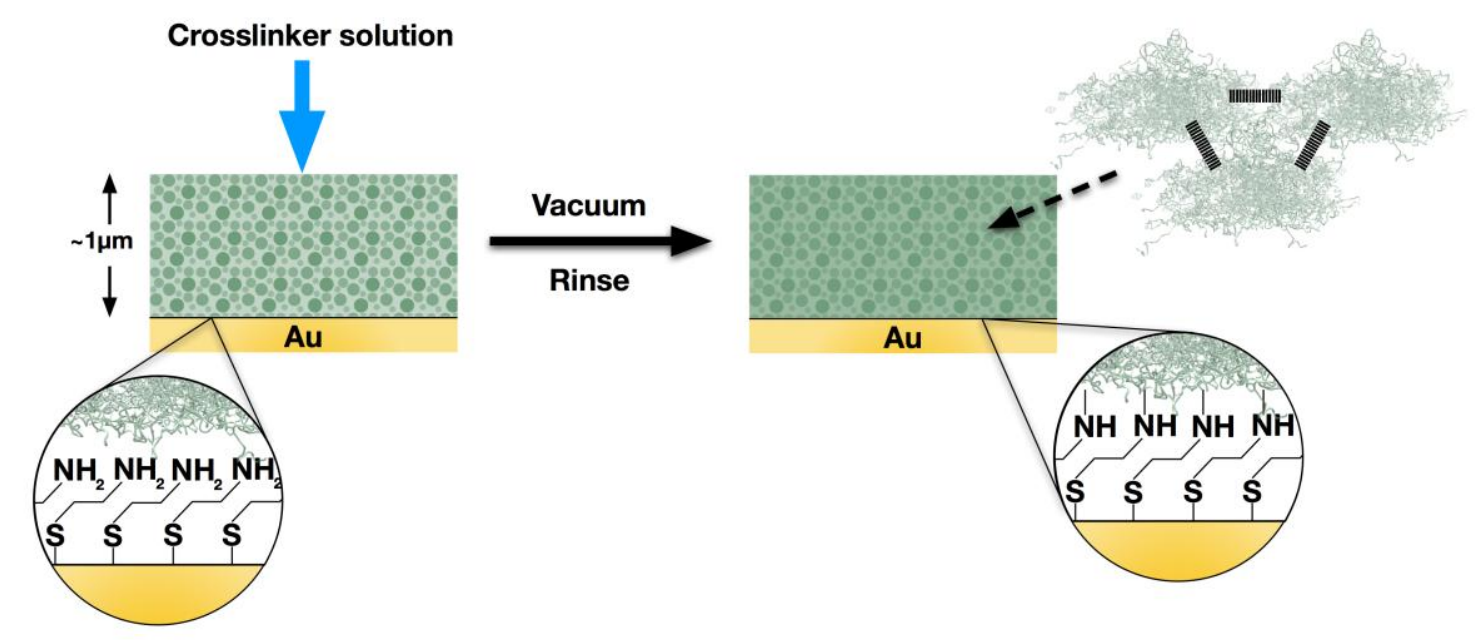

23

24

25

26

27

28

29

30

31

32

33

34

35

36

37

38

39

40

41

42

43

44

45

46

47

48

49

50

51

52

53

54

55

56

57

58

59

60

61

62

63 
Figure 2

(a)

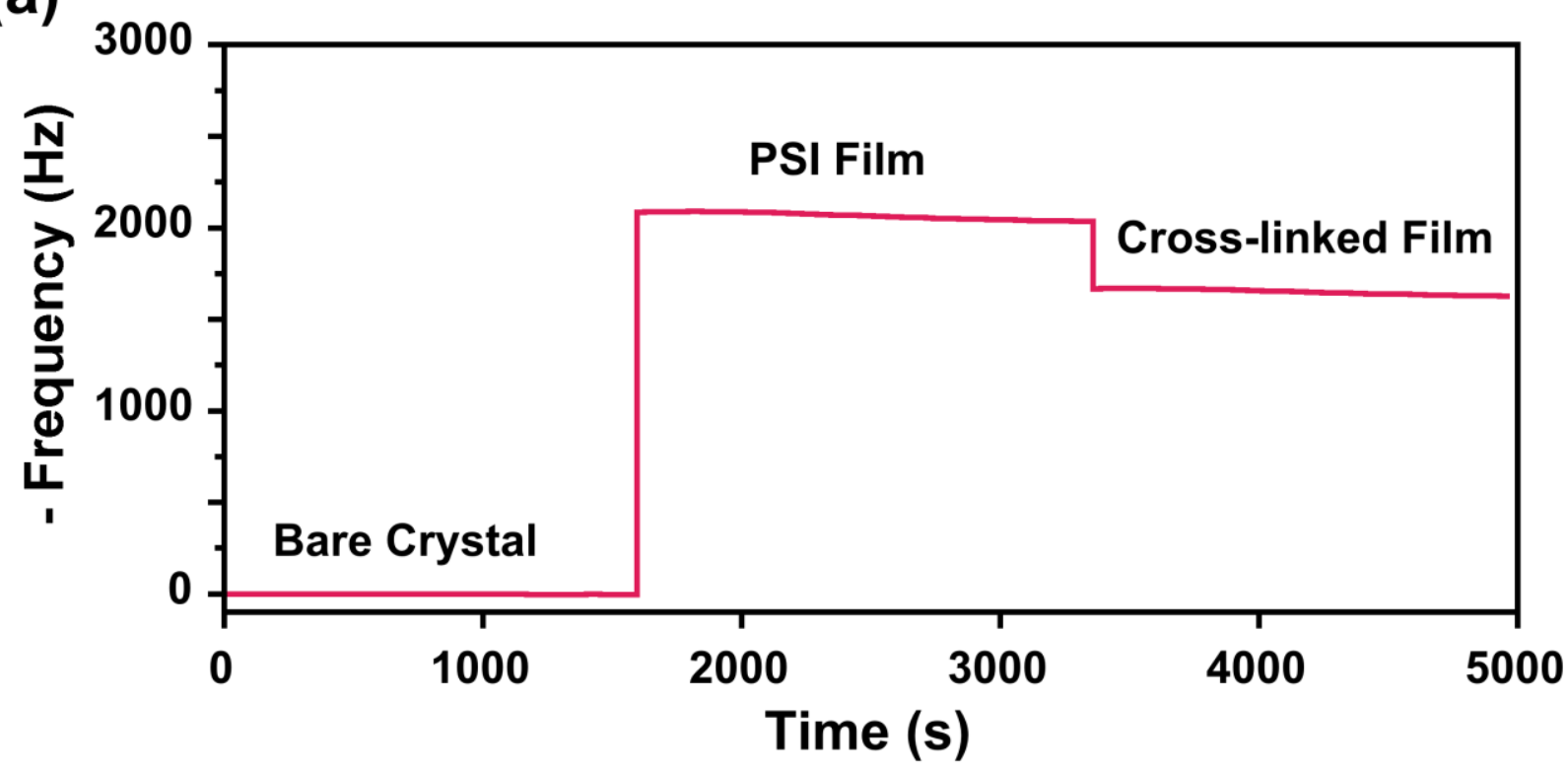

(b)

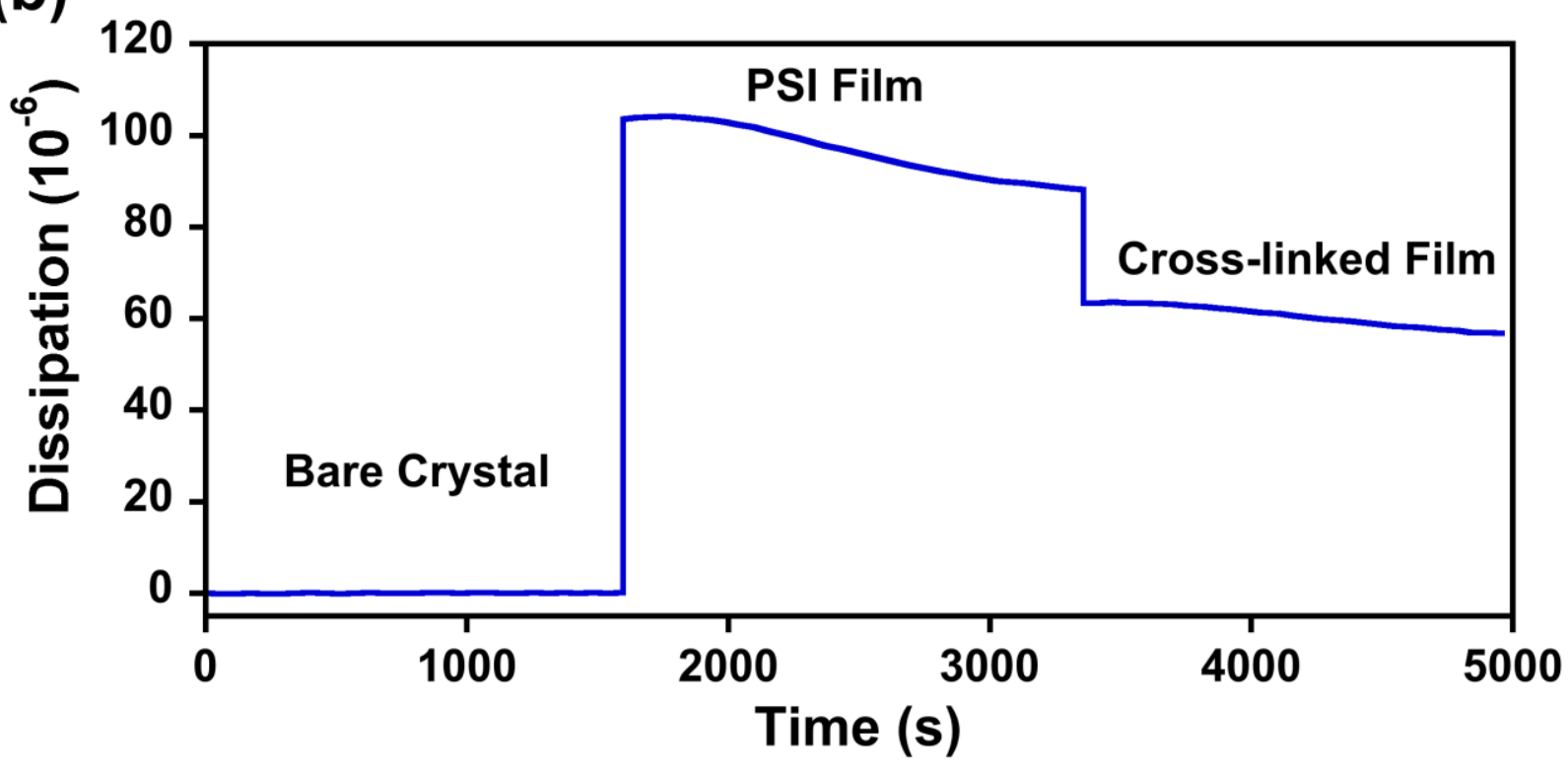


Figure 3

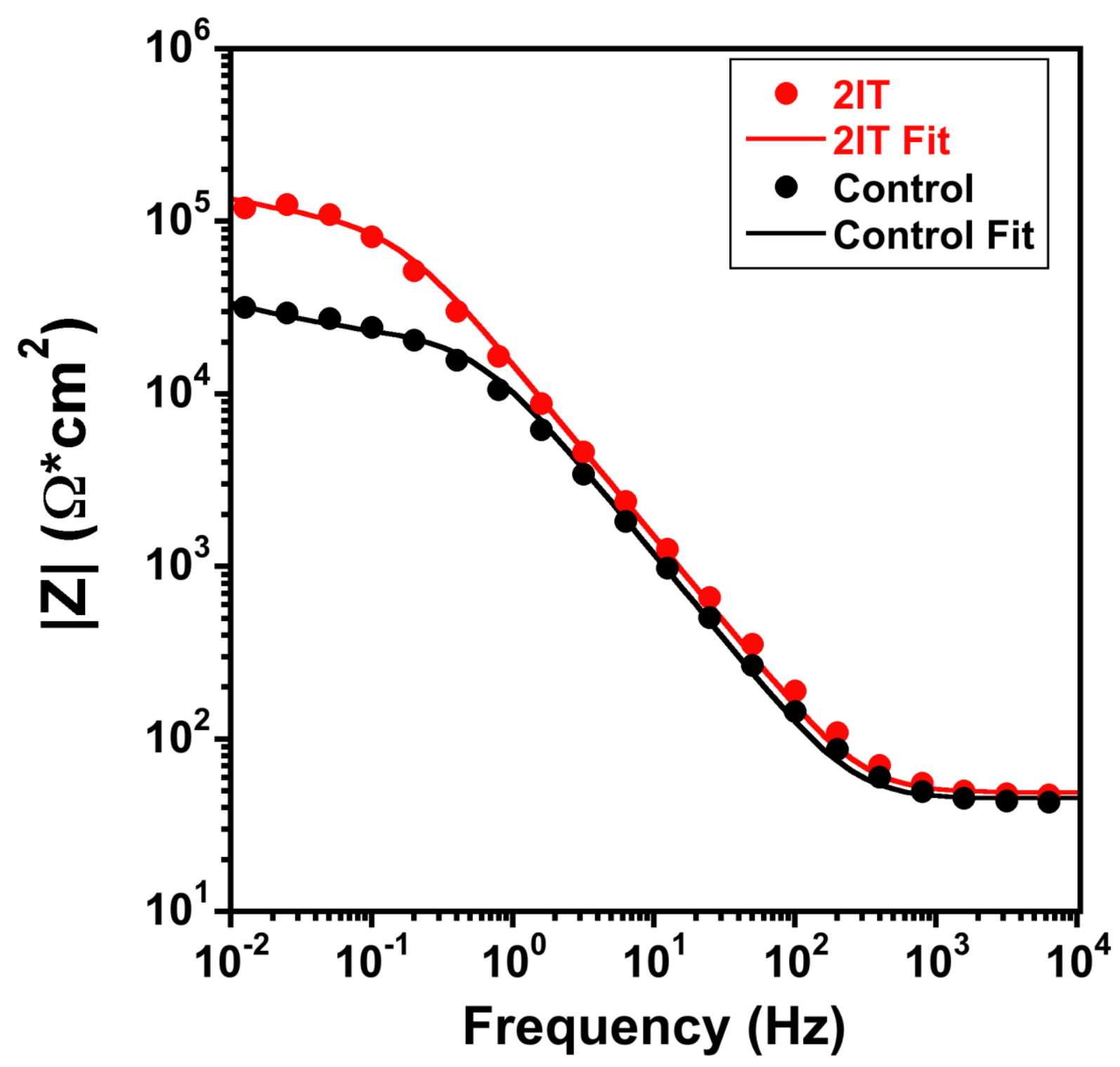


Figure 4

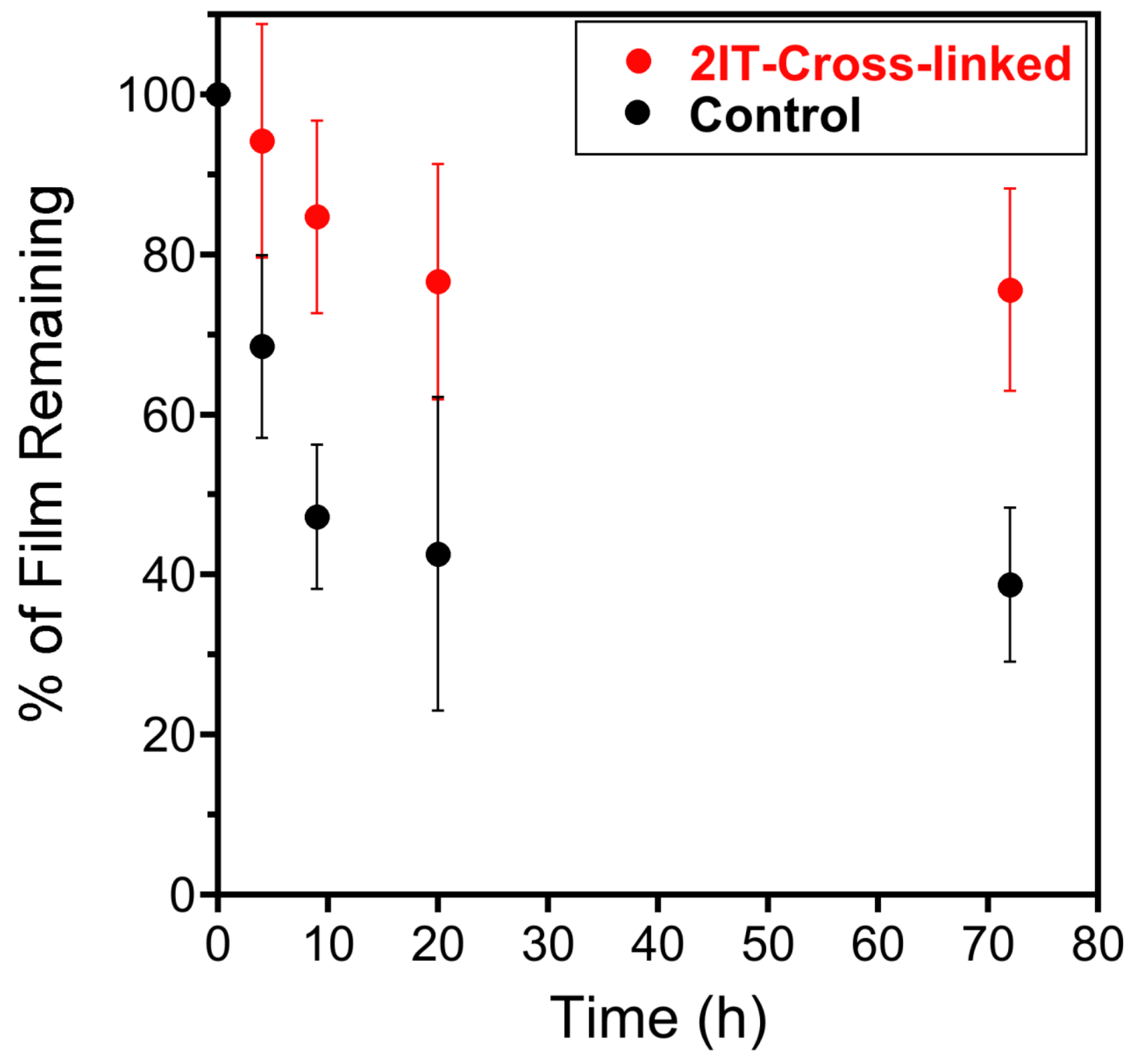

46

47

48

49

50

51

52

53

54

55

56

57

58

59

60

61

62 
Figure 5
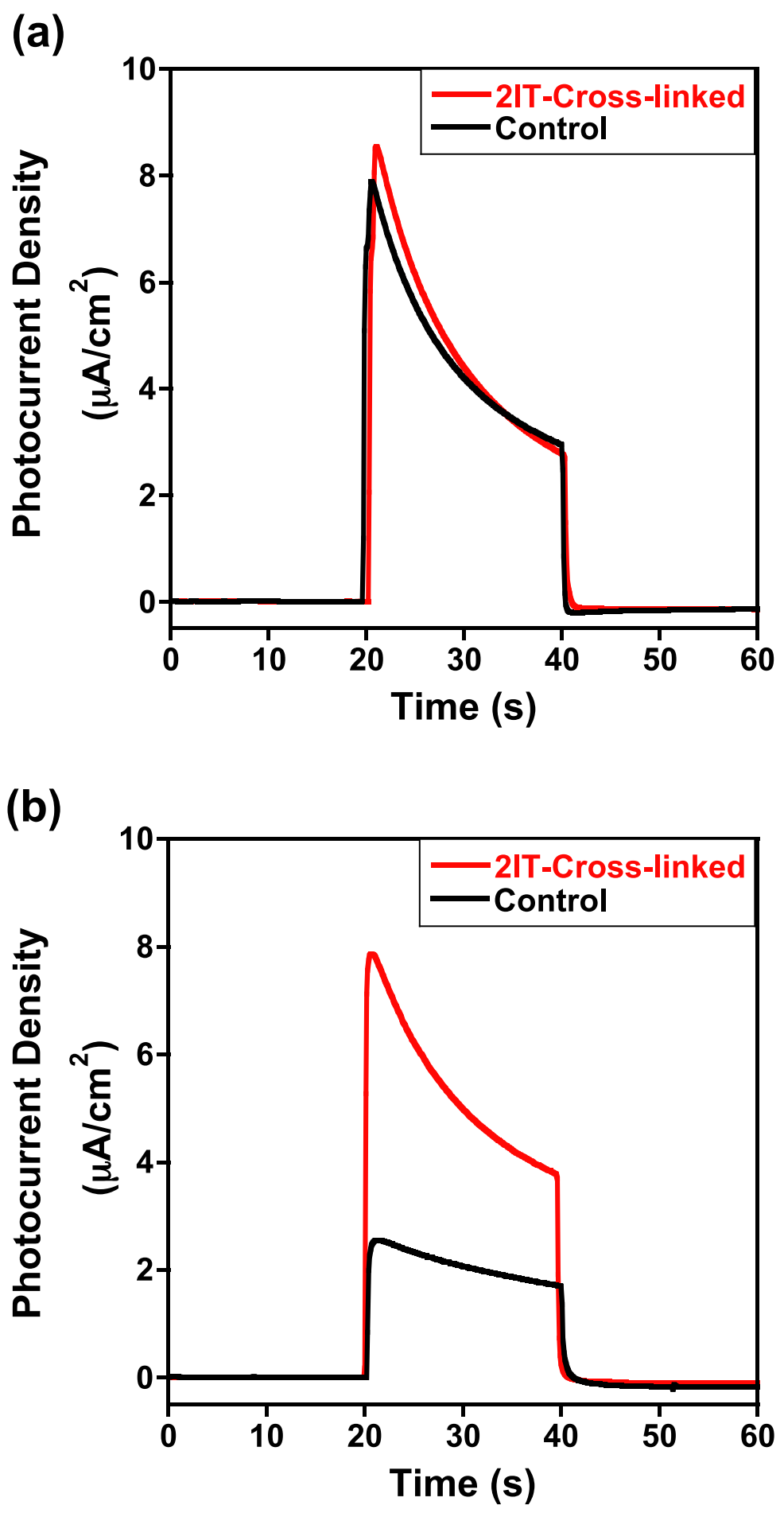